Documents pour l'histoire du français langue étrangère ou seconde

$24 \mid 1999$

Les auteurs classiques français dans l'enseignement du F.L.E. (18e et 19e siècles)

\title{
La culture française dans les études du «Real Colegio de Cirugía» de Cadix (1748-1800)
}

\section{Encarnación Medina Arjona}

\author{
C OpenEdition \\ Journals \\ Édition électronique \\ URL : https://journals.openedition.org/dhfles/3014 \\ DOI : $10.4000 /$ dhfles.3014 \\ ISSN : 2221-4038 \\ Éditeur \\ Société Internationale pour l'Histoire du Français Langue Étrangère ou Seconde \\ Édition imprimée \\ Date de publication : 1 décembre 1999 \\ Pagination : p.129-136 \\ ISSN : 0992-7654 \\ Référence électronique \\ Encarnación Medina Arjona, « La culture française dans les études du «Real Colegio de Cirugía» de \\ Cadix (1748-1800) », Documents pour I'histoire du français langue étrangère ou seconde [En ligne], 24 | \\ 1999, mis en ligne le 18 janvier 2015, consulté le 27 mai 2021. URL : http://journals.openedition.org/ \\ dhfles/3014 ; DOI : https://doi.org/10.4000/dhfles.3014
}

Ce document a été généré automatiquement le 27 mai 2021.

(c) SIHFLES 


\title{
La culture française dans les études $\mathrm{du}$ «Real Colegio de Cirugía» de Cadix (1748-1800)
}

\author{
Encarnación Medina Arjona
}

1 L'intérêt que l'on porte actuellement à l'histoire de l'enseignement des langues s'oriente de plus en plus vers l'acte quotidien: qui enseignait? à qui? où? dans quelles circonstances? et, bien sûr, avec quels outils? Par là s'explique notre démarche à la recherche de documents authentiques et significatifs, aidant à comprendre la réalité de l'apprentissage du français à Cadix au $18^{\mathrm{e}}$ siècle.

2 En 1748, le roi Ferdinand VI, montrant du zèle pour les sciences et étant conseillé par Juan Lacomba (chirurgien-major de l'Armée), fit créer le Real Colegio de Cirugía de la Armada de Cadix. Sous la protection du marquis de la Ensenada, le Collège commença avec Pedro Virgili.

3 Par décret royal de 1758, les études à suivre dans ce Collège aboutiraient au diplôme de bachelier ès-philosophie, degré obligatoire pour passer l'examen du Tribunal ProtoMedicato. Nous citons ici un texte de La France Littéraire ou Almanach des Beaux Arts de 1755 qui nous permet d'identifier le même principe législatif de l'instruction: «Pour être reçu à présent chirurgien à Paris, il faut être maître-ès-arts, et soutenir des thèses publiques» (25). Étant donné les similitudes précédentes, nous pensons aussi, pour Cadix, à des conditions d'admission au Collège plus ou moins pareilles qu'en France où «les élèves doivent avoir plus de quinze ans, [...] savoir lire et écrire, être exempt de tares physiques et savoir pratiquer la saignée.» (Rousselle: 43).

Entre ces conditions minimales de savoir et le besoin national de chirurgiens instruits, les responsables de la création du Collège lancent un pont monumental.

Il s'agit d'une riche bibliothèque digne de l'élan que ces études ressentent dans toute l'Europe. C'est un an après que les livres commencent à enrichir la collection (Gestido: 15), qui inquiète le Roi à partir même du moment de sa fondation. Or le fonds fut patiemment réuni par les professeurs du Collège grâce à une partie de leurs honoraires (aussi bien des officiers que des chirurgiens navigants) (Orozco: 101-115). Le 
responsable de cette bibliothèque devait être une personne d'une vaste et sérieuse instruction, extrêmement versée en bibliographie, en tant que chargé d'une collection si précieuse et actualisée. Ce bibliothécaire devait également maintenir une correspondance avec l'étranger (Ferrer: 95) et, d'après le décret royal, connaître parfaitement le français et le latin. C'est ainsi que les textes réglementaires dénotent un souci constant du pouvoir royal tout au long du $18^{\mathrm{e}}$ siècle à l'égard de la langue française.

6 La précieuse bibliothèque contenait non seulement des ouvrages médicaux et chirurgicaux, mais en plus un très important fonds relatif aux sciences de l'esprit. Le Collège désirait se procurer les classiques de la littérature et les dernières parutions françaises. En ce qui concerne le choix des livres, une recommandation de 1749 insistait sur l'intérêt d'acquérir tous les livres issus de l'Académie royale des sciences de Paris (ibid.: 344). Puis, c'est en 1752, lors d'un voyage de M. Manresa à Paris que celui-ci est chargé d'envoyer tous les livres parus cette année-là (voir Casas).

7 Nous venons de voir comment le besoin de lire les recueils chirurgicaux français, la contrainte des jeunes boursiers qui allaient puiser des connaissances en France, la nécessité d'entretenir une correspondance avec les Académies et les journaux scientifiques, l'usage du français (et le latin) qui redonnait son prestige à cette branche de la science, confirmaient le fondement de l'intérêt que le Collège portait à l'enseignement de la langue française. Cet apprentissage était à l'origine d'une bibliothèque à moitié française à raison de cinquante pour cent de ses titres.

8 Bartolomé-José Gallardo est peut-être le responsable du fonds disparu. Le renommé bibliophile fut chargé de choisir et de mettre à la disposition des députés de 1812 une sélection des livres de ce centre. Malgré l'opposition acharnée de M. Francisco Ameller y Clot (chirurgien-major), à l'époque directeur de l'établissement, la collection ne fut jamais rendue et le Collège subit une perte irréparable. C'est sur ces livres perdus que porte notre étude. En raison de cette perte nous avons cherché à savoir quels étaient les ouvrages utilisés à des fins didactiques dans l'apprentissage du français et nous nous proposons de faire connaitre leur contenu d'une façon générale. Il convenait d'abord de limiter l'étude dans le temps en fixant nos dates entre 1748 et 1800 .

9 Préalablement nous voulons situer ces manuels disparus dans le courant idéologique qui s'ouvrait à cette époque à l'organisation officielle de l'instruction en France. Étant donné qu'ils y étaient tous parus, nous pouvons les rattacher au contexte pédagogique de cette deuxième moitié du $18^{\mathrm{e}}$ siècle. En 1763 Louis-René de La Chalotais suggère dans son Essai d'éducation nationale ou Plan d'études pour la jeunesse que :

10 L'État doit soulager les maîtres, autant qu'il est possible, en faisant composer par des gens habiles des Livres élémentaires et classiques» et que «le plus grand service que les Sociétés Littéraires pussent rendre aux lettres, aux sciences et aux arts, était de faire des méthodes (La Chalotais: 34-44).

11 À propos de la littérature, le magistrat breton dira: «[...] il est honteux que dans une éducation de France on néglige la Littérature Française, comme si nous n'avions pas des modèles dans notre Langue» (71). Il s'ensuit que l'intérêt pour les méthodes nouvelles, pour les manuels utiles aux enfants, en un mot, l'organisation de l'apprentissage, va rapporter aux enseignants de ce siècle toute une collection de livres indispensable dans les bibliothèques de leurs établissements. L'ensemble documentaire que nous tenons $\mathrm{du}$ Real Colegio de Cirugía de Cadix, ainsi que les propos de La Chalotais -«Il paraît que par rapport aux vues d'éducation il y a dans le Public de l'Europe même, une espèce de 
fermentation qui doit naturellement faire de bons effets» (35)-, nous invite à penser que ces jeunes exclus de l'Université profitèrent de l'explosion didactique des manuels.

Cette institution où l'on formait les futurs chirurgiens disposait, selon un ordre chronologique, de l'Arte francés de Jean de Vayrac (1714) qui commence par une ample partie cherchant à résoudre des problèmes de prononciation et d'orthographe aux lecteurs étrangers (Bruña: 409-417). Cette grammaire contient un Traité de Poésie Française qui réserve des pages à Despréaux, à Corneille, aux odes de H. de la Mothe Houdart, ainsi qu'à une fable de Mademoiselle Bernard.

La bibliothèque comptait trois autres grammaires françaises. La première, A New French Grammarde Rogissard parut à Londres en 1738 (quoique l'édition inscrite dans les archives du Real Colegio date de 1759). Cet ouvrage bilingue français-anglais est plus approprié à la classe (voir Medina, 1997a) puisqu'il est conçu avec des chapitres destinés à pratiquer des phrases familières sur divers sujets, 46 dialogues familiers, 11 dialogues pour un niveau avancé et une collection de proverbes, entre autres. En ce qui concerne les textes littéraires ce sont les fables de La Fontaine qui l'emportent sur deux autres de Boileau, L'huître et les Plaideurs et Le Bûcheron et la Mort. Et finalement le conte, Les Oyes de Frère Philippe, tiré de La Fontaine et dédié «aux Belles».

Une deuxième grammaire, Les Vrais principes de la lecture, de l'orthographe, et de la prononciation française de Nicolas-Antoine Viard (première édition de 1763), édition revue et augmentée par M. Luneau de Boisjermain (1778), présente, à notre avis, le trait caractéristique des manuels, c'est-à-dire qu'il a été conçu (voir Medina, 1997b) pour être utile «aux enfants» (selon son sous-titre) et aux enseignants. Une "Instruction pour les personnes qui enseignent à lire» précède chaque chapitre du livre. Ces consignes indiquent des procédés à suivre: s'attarder sur tel sujet, s'attacher à faire lire tel Tableau, ménager un petit succès à l'élève, etc. Viard propose à la fin de la Première Partie quelques lectures suivies, imprimées à la fois en caractère romain et en italique (remarquons qu'il s'agit d'un livre «destiné à commencer l'éducation d'un Enfant»).

L'exemple de Racine y est présent avec Esther; avec le soin de choisir des passages où «l'idée de Dieu, et de son pouvoir sur toutes les créatures» s'y fait le plus évident, Viard ajoute également le Portrait de l'hypocrite et Stance sur la mort de Rousseau.

Les textes littéraires ne réapparaitront dans cet ouvrage qu'à la fin de la troisième Partie quand l'auteur s'arrête à considérer la «Ponctuation». Pour illustrer l'utilisation de la virgule et du point-virgule il recourt à l'Oraison funèbre de M. Le Vicomte de Turenne, par Fléchier; pour le point et le deux-points, il recourt à un texte de Mme de Sévigné; ainsi qu'aux Odes de Rousseau pour illustrer l'emploi du point d'interrogation et du point d'exclamation.

17 Une autre grammaire de ce fonds disparu avait pour titre Grammaire Française destinée au cours d'éducation des demoiselles, et des jeunes Messieurs qui ne veulent pas apprendre le latin, de M. Wandelaincourt (1782). L'auteur ne présente aucun texte littéraire ni aucune référence aux écrivains de l'époque classique.

Nous avons donc constaté à partir de ce passage eu revue des grammaires disparues de la bibliothèque que la contribution au savoir littéraire des étudiants, enseignants ou lecteurs en général était bien mince. Cependant ces chirurgiens lettrés manifestaient un esprit de lumières du point de vue de la science chirurgicale. Solidement implantée dans les sociétés savantes parisiennes, cette minorité «avancée» et «éclairée» participe activement au bouillonnement intellectuel des sociétés de pensée. Citons l'exemple de 
George Juan, commandant des Gardes de la Marine à Cadix, quand il apparaît dans La France Littéraire ou Almanach des Beaux Arts (1755), en tant que correspondant de l'Académie des sciences de Paris. Il se peut très bien qu'il y eût une prise de conscience des lacunes de l'enseignement reçu en ce qui concerne la littérature française. Pour corriger le manque, les Euvres diverses (1729J de Jean de la Fontaine ainsi que Les chefsd'œuvre dramatiques (1792) et Le théâtre (1728) de Corneille sont mentionnés dans les registres d'acquisitions du Real Colegio. Mais quelle était l'information littéraire qui leur venait de Paris à une époque de grande dualité entre les défenseurs en matière littéraire du siècle du goût et du siècle des Lumières?

Pour répondre à cette question il nous faudra voir Bibliothèque Française ou Histoire Littéraire de la France pour l'année 1727. Cet ouvrage fait part, au chapitre «Nouvelles Littéraires» (162), du livre de Pissot, Méthode très aisée pour apprendre l'orthographe par principes sans avoir étudié le Latin. Le commentaire qui suit est synthétique et assez révélateur: «On a tant écrit là-dessus, qu'on ne devrait point surcharger encore la librairie de ces livres inutiles» (162). Le tome IX présente également (242) De la Manière d'enseigner et d'étudier les Belles Lettres par M. Rollin. Ce dernier propose aux maîtres les grammaires de l'abbé Régnier et de M. Arnauld. En ce qui concerne la poésie, les tragédies Esther et Athalie de Racine y sont présentées comme modèles de lecture.

Le $18^{\mathrm{e}}$ siècle se présente donc comme une époque durant laquelle les méthodes d'enseignement jouissaient d'une importance amplifiée par rapport à l'intérêt que l'on porte aujourd'hui aux manuels dans les moyens d'information. Pour confirmer cette impression, on peut se référer aux «Observations adressées à M. Rollin, ancien Recteur et Professeur Royal, sur son Traité de la manière d'enseigner et d'étudier les Belles Lettres par M. Gibert ancien Recteur et Professeur de Rhétorique au Collège Mazarin» (tome X: 198), puis la réplique, «Lettre de M. Rollin à M. Gibert Ancien Recteur de l'Université, au sujet de ses Observations sur le Traité de la Manière d'enseigner et d'étudier les Belles Lettres» (234), et à nouveau la réfutation, «Réponse de M. Gibert à la Lettre de M. Rollin, Ancien Recteur de l'Université» (250). D'autres querelles sont reproduites dans la Bibliothèque Française, celle entretenue par Voltaire et Fontenelle, par exemple, à cause d'une critique du philosophe à propos de Mondes de Fontenelle. Signalons aussi que cette Histoire Littéraire de la France ajoute à la fin de chacun de ses volumes une liste de Livres de Médecine et de Chirurgie.

21 En ce temps de débat où la société est mise en cause par la philosophie, nous observons que la question de l'enseignement en général et celle de la littérature en particulier, à savoir le choix des textes de lecture, est le lieu où se joue une bataille idéologique importante. Les querelles parues dans les journaux nous en donnent la mesure. Le Collège de Cadix, qui s'enorgueillissait sans doute de la qualité des périodiques de sa collection, comptait Le Journal de Littérature, des Sciences et des Arts de l'abbé Grosier. Nous y retenons dans les «Idées Préliminaires» du tome I pour l'année 1779 les propos suivants:

Nos Philosophes ne sont pas seulement des Littérateurs unis et confédérés pour parvenir plus sûrement à la célébrité, ils forment encore une secte, qui distingue le zèle du prosélytisme et qui se croit seule chargée du dépôt de l'enseignement [...] (13). Puis quelques phrases annoncent la vente d'une gravure représentant «Voltaire couronné par Arlequin, le 30 Mars 1778, avec ces deux vers: Il est beau de la recevoir/ Quand c'est Arlequin qui la donne. A Paris chez les Marchands d'Estampes» (69). 
Le tome second rapporte la querelle surgie entre l'auteur du Dictionnaire des trois Siècles (que nous aurons l'occasion d'examiner plus bas) et M. L'abbé le $\mathrm{N}^{* * *}$. La critique de ce dictionnaire de littérature se présente sous le titre d'«Examen impartial de plusieurs observations sur la Littérature; Ouvrage où l'on fait l'éloge ou la critique des Auteurs Français, dont la lecture pourrait contribuer àformer ou à dépraver le goût des jeunes gens $[. .] ».(439)$.

Les Trois Siècles de la Littérature Française ou Tableau de l'Esprit de nos Écrivains, depuis François I, jusqu'en 1774 était l'un des ouvrages où les jeunes étudiants et les éminents professeurs de Cadix pouvaient puiser leurs connaissances sur les écrivains français. Il s'agit là d'un livre en forme de dictionnaire. Sabatier avait parsemé les différentes entrées de textes choisis pour les lecteurs, en observant la règle que lui-même avoue au début de la préface :

Quand nous avons donné la première Édition de cet Ouvrage [...] nous déclarions en un mot la guerre à la Philosophie. [...] Le Théâtre de la Littérature est envahi par trois sortes d'ennemis, qui la dégradent: une Philosophie tyrannique et inconséquente y suffoque ou corrompt les germes du talent, le faux goût y anéantit les vrais principes, une aveugle facilité à tout admirer achève d'en bannir l'émulation et de décourager le mérite. [...] Quel bouleversement dans les idées! Ce sont des Philosophes qui ont mis Lucain au-dessus de Virgile, Despreaux au-dessous de Quinault, la Motte à côté du grand Rousseau, Voltaire au-dessus de Corneille et de Racine, Perrault, Boindin et Terrasson audessus de tous les Écrivains du Siècle dernier. (III, XXIII. XXXII).

C'est donc avec l'intention de mettre au jour les travers littéraires, que l'auteur écrit en se référant à Despréaux: «M. De Voltaire est le premier qui ait ameuté contre lui tout le Corps des pygmées Littéraires qui combattent sous ses ordres» (49). Les jugements qu'Antoine Sabatier porte sur les écrits de Mme de La Fayette - « Avant elle, les Romans étaient l'ouvrage de l'imagination et jamais celui du sentiment. Elle a banni, la première, un héroïsme chimérique, et en a réduit la fiction à la peinture des mœurs, des caractères et des usages de la Société (107) » ou les éloges de l'esprit de Racine, ou du génie de Corneille, ainsi que de «connaissance profonde du cœur humain» (93) chez Molière, ne peuvent point être considérés comme impartiaux. Or l'auteur des Trois Siècles s'attache particulièrement à démolir d'Alembert: «Plusieurs Critiques respectables et éclairés nous ont reproché d'avoir traité avec trop d'indulgence ses Mélanges de Littérature: de n'avoir pas assez insisté sur les défauts de sa métaphysique souvent obscure, imperceptible, entortillée; sur les inégalités de son style, tantôt faible, tantôt plein de morgue, et presque toujours froid et bourgeois [...]» (10). Il essaie de ridiculiser Diderot: "Écrivain incorrect, Traducteur infidèle, Métaphysicien hardi; Moraliste dangereux, mauvais Géomètre, Physicien médiocre, Philosophe enthousiaste, Littérateur enfin qui a fait beaucoup d'ouvrages, sans qu'on puisse dire que nous ayons de lui un bon Livre » (59). Il cherche à déprécier le style de Beaumarchais: «Eugénie a eu un succès qu'on ne peut attribuer qu'au goût stupide du siècle pour les Comédies dolentes. Molière ne se serait pas attendu à se voir remplacer sur notre Théâtre, par des successeurs plaintifs, qui viendraient nous faire pleurer où nos Aïeux avaient trouvé tant de plaisir à rire. Le caractère de la Nation est-il changé? Non [...] ». Et, enfin, Sabatier cherche surtout à dénigrer Voltaire: «D'autres Littérateurs, aussi inconsidérés, n'ont pas craint d'élever la Muse tragique de M. De Voltaire au-dessus de celle de Corneille et de Racine. Comment n'ont-ils pas craint d'insulter à la crédulité publique [...]?» (153). 
Telles étaient donc les lectures et les grammaires qui s'offraient aux lecteurs de la bibliothèque du Real Colegio de Cirugía de Cadix. En Europe, la littérature médicochirurgicale et les Belles Lettres faisaient un ensemble. La tragédie, la grammaire et les mémoires d'hôpital formaient un tout dans les lectures des maîtres et de leurs élèves. C'est ce même savoir indivisible qui est disponible dans ce Collège. Le fonds à la disposition des jeunes étudiants était un espace considérable pour élargir leur culture.

En conclusion, s'appuyant sur l'enseignement en Europe, cet établissement cherche dans la connaissance de la langue française la clef de son perfectionnement et sa réputation grandissante. Le Collège dispensait une instruction moderne. Cependant, nous pensons que cet enseignement était déterminé par une relation de correspondance régulière avec les Académies scientifiques françaises et donc des connaissances enrichies dans leur dimension littéraire. Or ce tissu littéraire est composé de trois matières différentes. Les grammaires et leurs extraits de Boileau, Rousseau, Mme de Sévigné ou Racine apportent l'élément officiel venu de France; les œuvres de Corneille, La Fontaine et Racine y joignent le goût des enseignants espagnols; et, finalement, l'actualité de l'esprit mise au jour dans les débats des journaux où les textes classiques sont oubliés en faveur des polémiques du siècle.

\section{BIBLIOGRAPHIE}

BRUÑA, M. (1996): «La literatura en la enseñanza del francés en España en los siglos XVI al XVIII», Aproximaciones diversas al texto literario. Murcia, pp. 409-417.

CASAS, J. L. (1959): Contribución al estudio económico del Real Colegio de Cirujanos de la Armada de Cádiz, Cádiz.

FERRER, D. (1983): Historia del Real Colegio de Cirugía de la Armada de Cádiz, Cádiz.

GESTIDO, R. (1994): Una Biblioteca ilustrada gaditana, Cádiz.

LA CHALOTAIS, L. R. de (1985): Essai d'éducation nationale ou Plan d'études pour la jeunesse (1763), Maulévrier, pp. 34-44.

MEDINA, E. (1997a): «La utilización del tema Dans la classe en el manual de gramática francesa de Rogissard de 1738», Horizontes en la Didáctica de las Lenguas Aplicadas, Córdoba, (sous presse). MEDINA, E. (1997b): «Les Vrais principes de la lecture (1763) de Viard. Aproximación a un primer planteamiento didáctico de la fonética francesa», Tavira, Cádiz, (sous presse).

OROzCO, A. (1988): «Una Biblioteca singular en el Cádiz de Carlos III», El Reinado de Carlos III, Cádiz. ROUSSELLE, M. (1996): Médecins, chirurgiens et apothicaires français au Maroc (1577-1907), Jouve. 
AUTEUR

ENCARNACIÓN MEDINA ARJONA

Université de Cadix 